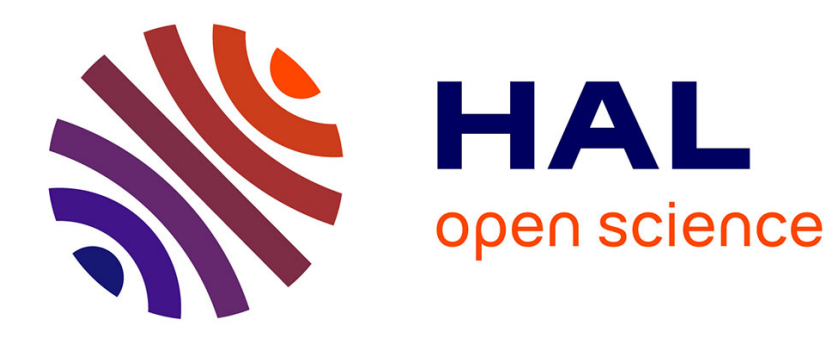

\title{
Pareto-efficiency and endogenous fertility: a simple model
}

\author{
Philippe Michel, Bertrand Wigniolle
}

\section{To cite this version:}

Philippe Michel, Bertrand Wigniolle. Pareto-efficiency and endogenous fertility: a simple model. Mathematical Population Studies, 2009, 16 (1), pp.36-59. 10.1080/08898480802619587 . halshs00306463

\section{HAL Id: halshs-00306463 \\ https://shs.hal.science/halshs-00306463}

Submitted on 25 Jul 2008

HAL is a multi-disciplinary open access archive for the deposit and dissemination of scientific research documents, whether they are published or not. The documents may come from teaching and research institutions in France or abroad, or from public or private research centers.
L'archive ouverte pluridisciplinaire HAL, est destinée au dépôt et à la diffusion de documents scientifiques de niveau recherche, publiés ou non, émanant des établissements d'enseignement et de recherche français ou étrangers, des laboratoires publics ou privés. 


\title{
Pareto-efficiency and endogenous fertility: a simple model*
}

\author{
Philippe Michel ${ }^{\dagger}$ and Bertrand Wigniolle
}

November 21, 2007

*The authors are grateful to an anonymous referee for useful suggestions and improvements.

${ }^{\dagger}$ GREQAM, Université de la Méditerranée. The current version of this paper was completed after Philippe Michel's sudden death. I want to express my deep sorrow for the loss of a close friend and an excellent economist, from whom I learned a lot over the years.

$\ddagger$ Centre d’Economie de la Sorbonne, Université de Paris I. Address: EUREQua, Maison des Sciences Economiques, 106-112, boulevard de l'hôpital, 75647 Paris, Cedex 13, France. Email : wignioll@univ-paris1.fr. 


\begin{abstract}
:
This article is devoted to the study of the Pareto-efficiency of the competitive equilibrium for a simple overlapping generations economy with endogenous fertility. For CES utility and production functions, it is proved that the economic properties of the economy are closely related to the two elasticities of substitution. First, the competitive equilibrium exists and is unique if the sum of the two elasticities is not smaller than one. Secondly, a set of parameters is provided such that the equilibrium is both in under-accumulation and inefficient. Thirdly, a sufficient condition is proved that ensures that an equilibrium converging in under-accumulation is Pareto-efficient: the sum of the two elasticities must not be greater than two.
\end{abstract}

Keywords: endogenous fertility, Pareto-efficiency.

JEL Classification: D9, J13, and D61. 


\section{Introduction}

This work is devoted to the study of the Pareto-efficiency of the competitive equilibrium for a simple overlapping generations economy with endogenous fertility.

Fertility is endogenous in our framework, and results from a choice made by each household. We assume that the number of children is an argument of the utility function of the parents and that each child entails a constant childrearing cost for his parents. Fertility behavior results from a rational choice, as consumption and saving choices. This framework is close to models used by Becker (1960), Willis (1973), Razin and Ben-Zion (1975) and Eckstein and Wolpin (1985). It leads to economic properties, which are relevant when compared to empirical facts, as is shown in of Birdsall's survey (1988).

We consider a simple framework where all individuals of the same generation are identical. We will refer to the well-being of one generation to designate the utility of each member of this generation. A competitive equilibrium is said to be Pareto-efficient if it is not possible to increase the well-being of one generation without decreasing the well-being of another.

The question of the Pareto-efficiency of the competitive equilibrium is well-known when fertility is exogenous. Considering the standard overlapping generations model à la Diamond (1965), a sufficient condition ensuring the Pareto-efficiency of the competitive equilibrium is that it converges towards a steady state such that the interest rate is greater than the growth rate. When there is no technical progress, the growth rate is equal to the growth rate of the population. When this sufficient condition holds, the competitive equilibrium is said to converge in under-accumulation. On the other hand, an equilibrium converging in over-accumulation (with a growth rate higher than the interest rate) is inefficient. It is possible to increase the utility of one or many generations without a fall in the utility of other generations (De la Croix and Michel (2002)).

These results have important consequences for economic policy. For example, in an economy converging in over-accumulation, it is possible to improve the welfare of all generations by inter-generational transfers from young to old people (a pay-as-you-go social security system, or the issuing of public debt, for instance).

What happens to these properties when fertility is endogenous? Before answering this question, the notion of Pareto-efficiency needs a new definition able to deal with the endogenous fertility framework. Indeed, the basic notion is defined for a given set of agents, while endogenous fertility requires comparing allocations associated with different sets of agents. We use a 
simple criterion based on particular assumptions. First, we consider the fertility level as a continuous number. Secondly, we only focus on symmetric allocations for all agents belonging to the same generation, and we call the symmetric allocations satisfying the resource constraint of the economy RCallocations (for "Representative-Consumer" allocations). In this context, it is possible to refer to the utility of a generation as the utility level reached by each agent belonging to this generation. Thirdly, we introduce a concept called "Representative-Consumer" efficiency (or RC-efficiency), which refers to an RC-allocation, for which no other allocation exists that would lead to a higher level of utility for all generations with a strict improvement for at least one generation.

This article adds several results to a first one on the subject (Michel and Wigniolle (2006)). In this former article, we showed that over-accumulation remains inefficient with endogenous fertility. We have also proved two main results. First, we exhibited an example in which an equilibrium in underaccumulation is not Pareto-efficient. Under-accumulation is no longer a sufficient condition ensuring efficiency. Secondly, we gave a general condition ensuring Pareto-efficiency: the limit value of the wage does not make it possible to finance a fertility level higher than the limit value of the interest factor.

In this article, we focus on a particular case: an economy with CES production and utility functions. In this framework, we are able to obtain additional results. We first give a condition that ensures the existence and uniqueness of the intertemporal equilibrium. This condition is that the sum of the two elasticities of substitution must not be smaller than one. Secondly, we provide a set of parameters such that the stationary state of the economy is both in under-accumulation and inefficient. This result generalizes the example given in Michel and Wigniolle (2006), and provides a better understanding of the causes of inefficiency. Thirdly, we prove a sufficient condition for the Pareto-efficiency of an equilibrium converging in under-accumulation: the sum of the two elasticities of substitution must not be greater than two. This result shows that a trade-off between the two substitution elasticities in production and utility exists, in order to ensure the efficiency of a competitive equilibrium converging in under-accumulation.

The intuition behind this last result is that an allocation can Paretoimprove a competitive equilibrium converging in under-accumulation, if it leads, in the long run, to a fertility level higher than the interest factor. Our result gives two arguments capable of precluding this increase in the fertility level. The first argument is the complementarity of production factors. Indeed, the increase in fertility is linked to a fall in capital accumulation. If capital and labor are substitutable, this change is not too detrimental to 
production; if capital and labor are complementary, however, it can lead to a significant drop in resources. The second argument is the complementarity of consumption and fertility in the utility function. If consumption and fertility levels are highly substitutable, an increase in fertility allows for a substantial reduction in consumption, which is compatible with a constant level of utility. When they are complementary, however, the admissible fall in consumption is low.

Our results can be compared with existing literature.

Two recent studies are concerned with the definition of Pareto-efficiency criteria in an endogenous fertility framework. Conde-Ruiz, Giménez and Perez-Nievas (2004) use a slightly more general framework, where technical progress can affect both the production technology and the children cost function. They use the same criterion of efficiency which they call "Millian efficiency". They undertake a detailed analysis of efficiency criteria and provide several conditions for Millian-efficiency. With respect to their work, this article focuses on a less general framework, which allows us to provide a sufficient condition for the efficiency of an equilibrium in under-accumulation that directly involves the parameters of the production and utility functions.

Golosov, Jones and Tertilt (2004) provide a general analysis of Paretoefficiency criteria in a framework in which fertility level is a discrete variable and in the presence of intragenerational heterogeneity. This article appears as the more general and thorough on the question of Pareto-efficiency. But its results cannot be applied to a large range of simple macroeconomic models with endogenous fertility, particularly to models in which fertility is a continuous variable.

Section Two presents the model. Section Three studies the intertemporal equilibrium. Section Four introduces the main definitions concerning Paretoefficiency. Section Five gives a sufficient condition ensuring the Paretoefficiency of a competitive equilibrium converging in under-accumulation. Section Six concludes.

\section{The model}

\subsection{Consumers}

We make use of an overlapping generations model. Each individual lives for two periods. He or she inelastically supplies one unit of labor during his/her youth and retires in old age. During their first period of life, agents work, consume, save and choose their number of children. Children entail a cost 
for their parents. In their second period of life, agents consume the proceeds of theirs savings.

At each date $t$, the new generation is populated with a number $N_{t}$ of identical agents. Each agent is endowed with an intertemporal CES utility function

$$
U_{t}=\left(c_{t}^{\frac{\rho-1}{\rho}}+\beta d_{t+1}^{\frac{\rho-1}{\rho}}+\gamma m_{t}^{\frac{\rho-1}{\rho}}\right)^{\frac{\rho}{\rho-1}}
$$

where $\rho$, the elasticity of substitution, is such that $\rho>0$ and $\rho \neq 1$. $c_{t}$ and $d_{t+1}$ are respectively the consumptions of good during youth and old age, and $m_{t}$ is the number of children ${ }^{1}$. For the limit case $\rho=1$, we consider the log-linear function

$$
U_{t}=\gamma_{1} \ln c_{t}+\gamma_{2} \ln d_{t+1}+\gamma_{3} \ln m_{t}
$$

with $\sum_{i=1}^{3} \gamma_{i}=1$.

The number of agents of generation $t+1$ is equal to

$$
N_{t+1}=m_{t} N_{t}
$$

The first period budget constraint faced by a generation $t$ agent is

$$
c_{t}+\phi m_{t}+s_{t}=w_{t}
$$

where $w_{t}$ is the wage earned during this period and $s_{t}$ the savings. Each child entails a constant educational cost $\phi$.

Savings are invested in capital and yield a gross return in $t+1$, equal to $R_{t+1}$. The second period budget constraint is

$$
d_{t+1}=R_{t+1} s_{t}
$$

From (4) and (5), the lifetime budget constraint can be stated as

$$
c_{t}+\frac{d_{t+1}}{R_{t+1}}+\phi m_{t}=w_{t}
$$

Maximizing the utility functions (1 or 2) under the constraint (6) leads to the following demand functions

$$
\begin{aligned}
c_{t} & =w_{t} \gamma_{1}\left(R_{t+1}\right) \\
\phi m_{t} & =w_{t} \gamma_{3}\left(R_{t+1}\right) \\
s_{t} & =d_{t} / R_{t+1}=w_{t} \gamma_{2}\left(R_{t+1}\right) .
\end{aligned}
$$

\footnotetext{
${ }^{1}$ As usual, we consider $m_{t}$ as a continuous variable. This assumption is consistent with a model which assumes one representative agent per generation.
} 
When the utility function is given by: (1), the functions $\gamma_{i}(R)$ are

$$
\begin{aligned}
\gamma_{1}(R) & =\frac{1}{1+\beta^{\rho} R^{\rho-1}+\gamma^{\rho} \phi^{1-\rho}}, \\
\gamma_{2}(R) & =\frac{\beta^{\rho} R^{\rho-1}}{1+\beta^{\rho} R^{\rho-1}+\gamma^{\rho} \phi^{1-\rho}}, \\
\gamma_{3}(R) & =\frac{\gamma^{\rho} \phi^{1-\rho}}{1+\beta^{\rho} R^{\rho-1}+\gamma^{\rho} \phi^{1-\rho}} .
\end{aligned}
$$

When the utility function is given by: $(2)$, the functions $\gamma_{i}(R)$ are constant and equal to $\gamma_{i}$.

At date $t=0$, the $N_{-1}$ old agents consume the proceeds of their savings: $d_{0}=R_{0} s_{-1} . N_{-1}, s_{-1}$ and $N_{0}$ come from past choices and are given.

\section{$2.2 \quad$ Firms}

We assume that at each period $t$ one competitive firm exists, which produces $Y_{t}$ quantity of good, according to a CES production function with two inputs (capital: $K_{t}$; labor: $L_{t}$ )

$$
F(K, L)=A\left(K^{\frac{\tau-1}{\tau}}+b L^{\frac{\tau-1}{\tau}}\right)^{\frac{\tau}{\tau-1}}
$$

with $\tau>0$ and $\tau \neq 1$. When the elasticity of substitution $\tau$ is equal to 1 , the production function is Cobb-Douglas

$$
F(K, L)=A K^{\alpha} L^{1-\alpha} .
$$

The capital fully depreciates within the production period.

The profit maximization of the firm gives

$$
\begin{aligned}
& w_{t}=F_{L}^{\prime}\left(K_{t}, L_{t}\right) \equiv w\left(K_{t} / L_{t}\right), \\
& R_{t}=F_{K}^{\prime}\left(K_{t}, L_{t}\right) \equiv R\left(K_{t} / L_{t}\right) .
\end{aligned}
$$

When the production function is given by (13), $w$ and $R$ have the following expression

$$
\begin{aligned}
& w(x)=A b\left(x^{\frac{\tau-1}{\tau}}+b\right)^{\frac{1}{\tau-1}}, \\
& R(x)=A\left(1+b x^{\frac{-(\tau-1)}{\tau}}\right)^{\frac{1}{\tau-1}} .
\end{aligned}
$$

When it is given by (14), we obtain

$$
\begin{aligned}
& w(x)=A(1-\alpha) x^{\alpha}, \\
& R(x)=A \alpha x^{\alpha-1} .
\end{aligned}
$$




\section{The intertemporal equilibrium}

\subsection{Equilibrium characterization}

The initial conditions are the following. At period $t=0, N_{-1}$ old agents and $N_{0}$ young agents are living. The old agents hold equal shares of the capital stock $K_{0}$. This stock comes from their past savings behavior: $K_{0}=N_{-1} s_{-1}$. The consumption of the old agents is

$$
d_{0}=R_{0} K_{0} / N_{-1}
$$

Along an intertemporal equilibrium, all markets are balanced.

The labor market is balanced at each period: $L_{t}=N_{t}$, with $N_{t}$ following the relation $N_{t+1}=m_{t} N_{t}$.

Prices result from the firms' behavior at equilibrium

$$
R_{t}=R\left(k_{t}\right) \text { and } w_{t}=w\left(k_{t}\right) \text {, with: } k_{t} \equiv K_{t} / N_{t} .
$$

The commodity market is balanced, or

$$
F\left(K_{t}, N_{t}\right)=N_{t} c_{t}+N_{t-1} d_{t}+K_{t+1}+N_{t} \phi m_{t} .
$$

Taking consumers' budget constraints into account, this last relation is equivalent to

$$
K_{t+1}=N_{t} s_{t} \Leftrightarrow m_{t} k_{t+1}=s_{t} .
$$

Finally, using optimal behaviors and equilibrium conditions, it is possible to give the general definition of the intertemporal equilibrium:

Definition 1 Starting from the positive initial conditions $N_{-1}, N_{0}, K_{0}$ and $d_{0}=R_{0} K_{0} / N_{-1}$, an intertemporal equilibrium is a sequence $\left(K_{t}, N_{t}, c_{t}, d_{t}, m_{t}\right)_{t \geq 0}$, which satisfies

$$
\begin{aligned}
c_{t}+\frac{d_{t+1}}{R_{t+1}}+\phi m_{t} & =w_{t}, \\
U_{c_{t}} & =R_{t+1} U_{d_{t+1}}=\frac{U_{m_{t}}}{\phi}, \\
N_{t+1} & =m_{t} N_{t}, \\
F\left(K_{t}, N_{t}\right) & =N_{t} c_{t}+N_{t-1} d_{t}+K_{t+1}+N_{t} \phi m_{t}, \\
\text { with } R_{t} & =R\left(k_{t}\right) \text { and } w_{t}=w\left(k_{t}\right), \text { where: } k_{t}=K_{t} / N_{t} .
\end{aligned}
$$

This general definition allows us to encompass the different choices of utility and production functions. When it is possible, it will generally be more simple to reason with general functions. 


\subsection{Existence and convergence of intertemporal equi- libria}

In this part, we study the existence of the intertemporal equilibrium and its convergence toward a steady state.

Using equations (8), (9), (22) and (24), an intertemporal equilibrium is characterized by a sequence $\left(k_{t}\right)_{t \geq 0}$ starting from a given initial value $k_{0}=$ $K_{0} / N_{0}$, and following the equation

$$
k_{t+1}=\frac{s_{t}}{m_{t}}=\frac{\phi \gamma_{2}\left[R\left(k_{t+1}\right)\right]}{\gamma_{3}\left[R\left(k_{t+1}\right)\right]} .
$$

This equation only depends on $k_{t+1}$ and not on $k_{t}$. This property is obtained as the utility function is homothetic. As $s_{t}$ and $m_{t}$ are proportional to $w_{t}$, $k_{t+1}$ no longer depends on $w_{t}$. The two adjustments in savings and fertility lead to a constant ratio $K_{t+1} / N_{t+1}$ in one period.

There is existence and uniqueness of the intertemporal equilibrium if this equation has a unique solution $k_{t+1}=\bar{k}$. In this case, the economy reaches its stationary state $\bar{k}$ in one period. $\bar{k}$ is defined by

$$
\bar{k} \gamma_{3}[R(\bar{k})]=\phi \gamma_{2}[R(\bar{k})] .
$$

If $\forall t \geq 1, k_{t}=\bar{k}$, we obtain that the variables $R_{t}=R\left(k_{t}\right), w_{t}=w\left(k_{t}\right)$, $c_{t}, d_{t}$ and $m_{t}$ are also equal to constant values $\bar{R}, \bar{w}, \bar{c}, \bar{d}$, and $\bar{m}$. As in Diamond's model with exogenous fertility, we say that the steady state is in under-accumulation if $\bar{R}>\bar{m}$, and in over-accumulation if $\bar{R}<\bar{m}$.

It remains to study the conditions ensuring a unique solution for equation (31). We successively consider the case of a log-linear utility and the case of a CES utility.

When the utility is log-linear (given by (2)), the economy converges to the steady state in one period

$$
\bar{k}=\frac{\phi \gamma_{2}}{\gamma_{3}}
$$

When a CES utility function is assumed (given by (1)), the intertemporal equilibrium exists and is unique if the expression $k R(k)^{1-\rho}$ increases from 0 to $+\infty$ when $k$ goes from 0 to $+\infty$. Indeed, $\bar{k}$ is such that

$$
\bar{k} \gamma^{\rho} \phi^{1-\rho}=\phi \beta^{\rho} R(\bar{k})^{\rho-1},
$$

or

$$
\bar{k} R(\bar{k})^{1-\rho}=\phi^{\rho} \beta^{\rho} \gamma^{-\rho} .
$$


For the CES production function (13), $\bar{k}$ exists and is unique when $\tau+\rho \geq$ 1. Indeed, it is the unique solution of the equation

$$
k\left(1+b k^{-\frac{\tau-1}{\tau}}\right)^{-\frac{\rho-1}{\tau-1}}=A^{\rho-1} \phi^{\rho} \beta^{\rho} \gamma^{-\rho} .
$$

For the Cobb-Douglas production function (14), there is no condition on $\rho$ for the existence and uniqueness of the intertemporal equilibrium and $\bar{k}$ is equal to

$$
\bar{k}=\left((\alpha A)^{\rho-1} \phi^{\rho} \beta^{\rho} \gamma^{-\rho}\right)^{\frac{1}{1+(1-\alpha)(\rho-1)}} .
$$

Finally, we can summarize these results by the following proposition:

Proposition 1 If $\tau+\rho \geq 0$, the intertemporal equilibrium exists and is unique. The economy reaches its stationary state in one period.

\section{Efficient allocations and endogenous fertil- ity}

In the Diamond [1965] model with exogenous fertility, the Pareto-optimality properties of the competitive equilibrium are well-known. An intertemporal equilibrium converging to a stationary state in under-accumulation is Paretooptimal. On the other hand, an intertemporal equilibrium converging to a stationary state in over-accumulation is not Pareto-optimal. De la Croix and Michel (2002) provide a careful presentation of these results.

In this section, we show that the concept of Pareto-optimality needs a new definition in a framework of endogenous fertility. We first introduce the basic notion of feasible allocations. This allows us to introduce efficiency.

\subsection{Feasible allocations with representative consumers}

We define the notion of a feasible allocation with representative consumers as an intertemporal allocation that satisfies the resource constraints of the economy.

Definition 2 (RC-allocation) ${ }^{2}$ A feasible allocation with representative consumers (or RC-allocation) is a sequence $\left(K_{t}, N_{t}, c_{t}, d_{t}, m_{t}\right)_{t \geq 0}$ of positive variables that satisfy $\forall t \geq 0$

$$
\begin{aligned}
& F\left(K_{t}, N_{t}\right)=K_{t+1}+N_{t} c_{t}+N_{t-1} d_{t}+N_{t+1} \phi, \\
& N_{t+1}=m_{t} N_{t} .
\end{aligned}
$$

\footnotetext{
${ }^{2}$ An RC-allocation means a feasible allocation for Representative Consumers.
} 
For a competitive equilibrium, the initial conditions $N_{-1}, N_{0}, K_{0}$ and $d_{0}=$ $R_{0} K_{0} / N_{-1}$ are given. Following definition 2, an intertemporal equilibrium is a particular case of feasible allocation, which moreover satisfies equations (7), (8) and (9), with (22). From assumption 1, if $K_{0}, N_{0}$ and $N_{-1}$ are positive, all the variables $\left(K_{t}, N_{t}, c_{t}, d_{t}, m_{t}\right)_{t \geq 0}$ will be positive.

Remark 1 To simplify the presentation, we will always consider the four initial quantities $\left(N_{-1}, N_{0}, K_{0}, d_{0}\right)$ as given. It will be hereafter implicit that all feasible allocations or competitive equilibria considered will start from these same initial conditions, even if they are not mentioned.

A new characterization of feasible RC-allocations will be useful in what follows. In equation (37), $c_{t}$ and $m_{t}$ are chosen by generation $t$ agents when $d_{t}$ is determined by generation $t-1$ agents. By a simple change in variables, we can introduce a new definition of feasible allocations, in which all variables are decided by the same generation.

Lemma 1 (Characterizations of feasible RC-allocations) 1. Any feasible $R C$-allocation can be characterized as a sequence $\left(y_{t}, N_{t}, c_{t}, d_{t}\right.$, $\left.m_{t}\right)_{t \geq 0}$ of positive variables starting from the initial condition $y_{0}=$ $F\left(K_{0} / N_{0}, 1\right)-N_{-1} d_{0} / N_{0}$, and satisfying $\forall t \geq 0$,

$$
\begin{aligned}
& m_{t} y_{t+1}=F\left(y_{t}-c_{t}-\phi m_{t}, m_{t}\right)-d_{t+1}, \\
& N_{t+1}=m_{t} N_{t}, \\
& y_{t}>c_{t}+\phi m_{t} .
\end{aligned}
$$

Proof: This result is immediately obtained by a simple change in variables in the resource constraint of the economy

$$
y_{t}=K_{t+1} / N_{t}+c_{t}+\phi m_{t} .
$$

Remark 2 Let $\left(\bar{K}_{t}, \bar{N}_{t}, \bar{c}_{t}, \bar{d}_{t}, \bar{m}_{t}\right)_{t>0}$ be an intertemporal equilibrium. Along such a trajectory, the new variable $y_{t}$ is equal to the wage

$$
\bar{y}_{t}=\bar{w}_{t}=F_{L}^{\prime}\left(\bar{K}_{t}, \bar{N}_{t}\right) \text {. }
$$




\subsection{Efficiency of RC-allocations}

Pareto-efficiency is usually defined for a given set of agents. The model with endogenous fertility introduces a new difficulty, because the set of agents is the result of individual behaviors. We only consider the case of identical agents within each generation. In order to compare RC-feasible allocations, we use the main definition introduced in Michel and Wigniolle (2006).

Definition 3 (RC-dominance) ${ }^{3}$ Let $\left(K_{t}^{i}, N_{t}^{i}, c_{t}^{i}, d_{t}^{i}, m_{t}^{i}\right)_{t \geq 0}$ for $i=1,2$ be two feasible RC-allocations. Allocation 1 is said to $R C$-dominate allocation 2 if it leads to a higher level of utility for all generations, with a strict improvement for (at least) one generation. Formally,

$$
\begin{aligned}
\forall t & \geq 0, U\left(c_{t}^{1}, d_{t+1}^{1}, m_{t}^{1}\right) \geq U\left(c_{t}^{2}, d_{t+1}^{2}, m_{t}^{2}\right), \\
\exists t_{0} & \geq 0, \text { such that: } U\left(c_{t_{0}}^{1}, d_{t_{0}+1}^{1}, m_{t_{0}}^{1}\right)>U\left(c_{t_{0}}^{2}, d_{t_{0}+1}^{2}, m_{t_{0}}^{2}\right) .
\end{aligned}
$$

From the notion of RC-dominance, a definition of efficiency follows:

Definition $4 A n R C$-allocation is said to be $R C$-efficient, if there does not exist another $R C$-allocation that $R C$-dominates it.

Conde-Ruiz, Giménez and Perez-Nievas (2004) use the same concept of efficiency which they call "Millian efficiency", and they provide a general discussion of its relevance.

Michel and Wigniolle (2006) discuss another notion, which is obtained in adding to the definition of RC-dominance the constraints

$$
\forall t \geq 0, m_{t}^{1} \geq m_{t}^{2}
$$

For this last notion, an allocation can dominate another one only if it includes at least an equal number of children in each period. On the other hand, RCdominance allows to improve the utility level of one (or many) generation(s) to the detriment of the size of one (or many) generation(s). In the current article, we will neglect this second notion, as it does not allow us to obtain more results. Indeed, it will appear that these two notions lead to the same classification of competitive equilibria. The reason behind this result is that non-RC-efficient equilibria are dominated by allocations that include a higher level of fertility.

\footnotetext{
${ }^{3} \mathrm{RC}$-dominance means dominance for Representative Consumers.
} 


\section{$5 \quad$ Efficiency of the intertemporal equilibrium}

In this section, we focus on the question of the Pareto-efficiency of the competitive equilibrium with endogenous fertility, using our framework with CES utility and production functions. First, we provide a set of parameters such that the competitive equilibrium is both in under-accumulation and inefficient. This result enlarges the simple example given in Michel and Wigniolle (2006). Secondly, we give a sufficient condition ensuring that a competitive equilibrium converging in under-accumulation is Pareto-efficient. This condition only involves the two elasticities of substitution of the production and utility functions.

\subsection{Under-accumulation does not imply efficiency}

The following proposition gives a set of parameters such that the stationary state of our CES economy is both in under-accumulation and inefficient. The following notations will be useful

$$
\begin{aligned}
\tilde{\rho} & \equiv \frac{\ln (A \phi)}{\ln \left(\frac{\beta A \phi}{\gamma}\right)} \\
A_{1} & \equiv \frac{4 \phi \beta^{\rho} A^{\rho-1}\left(1+\gamma^{\rho} \phi^{1-\rho}+\beta^{\rho} A^{\rho-1}\right)}{\left(\gamma^{\rho} \phi^{1-\rho}+\beta^{\rho} A^{\rho-1}\right)^{2}} \\
A_{2} & \equiv \frac{1+\gamma^{\rho} \phi^{1-\rho}+\beta^{\rho} A^{\rho-1}}{\gamma^{\rho} \phi^{-\rho}}
\end{aligned}
$$

Proposition 2 Let us assume that one of these 3 conditions holds

$$
\begin{aligned}
\frac{\gamma}{\beta} & <A \phi<1 \\
\text { or } \frac{\gamma}{\beta} & <1<A \phi \text { and } \rho>\tilde{\rho}, \\
\text { or } A \phi & <1<\frac{\gamma}{\beta} \text { and } \rho<\tilde{\rho},
\end{aligned}
$$

then we have $A_{1}<A_{2}$, and, for each value of $b$ such that

$$
A_{1}<b<A_{2},
$$

the competitive stationary equilibrium is both in under-accumulation and inefficient for sufficiently high $\tau$. 
Proof: see Appendix 1.

As shown in Appendix 1, the proof is obtained by building a RC-allocation that gives the same consumption levels, and a higher fertility level, with respect to the competitive equilibrium. The fertility level rises in such a way that it becomes higher than the interest factor. The result is obtained taking the limit case $\tau \rightarrow+\infty$. At the limit, the interest factor becomes constant and independent of the capital-labor ratio $k$. In this case, it is possible to devote more resources to children and fewer resources to capital accumulation in such a way that the fertility level becomes higher than the interest factor.

To obtain this result, the initial stationary equilibrium must be such that the share of the wage devoted to savings $\gamma_{2}[R(\bar{k})]$ is higher than the share devoted to children $\gamma_{3}[R(\bar{k})]$. This property can be obtained under various values of the different parameters. At the limit when $\tau \rightarrow+\infty$, this property occurs if one among the three conditions (47), (48) or (49) holds. If (47) is satisfied, no condition on the elasticity of substitution of the utility function $\rho$ is needed. Condition (48) requires a sufficiently high value of $\rho$ and (49) a sufficiently low value of $\rho$.

\subsection{A sufficient condition for the efficiency of an in- tertemporal equilibrium}

In standard microeconomics, for a finite number of agents endowed with a utility function, a sufficient condition for an allocation to be Pareto-optimal is that this allocation maximizes a weighted sum (with positive weights) of each agent's utilities. In our model, in which we have considered an infinite number of agents of different generations, this property always holds, as illustrated in the following lemma.

Lemma 2 Let $\left(\bar{y}_{t}, \bar{N}_{t}, \bar{c}_{t}, \bar{d}_{t}, \bar{m}_{t}\right)_{t \geq 0}$ be a feasible $R C$-allocation. Let $\left(\beta_{t}\right)$ be a sequence, such that $\forall t, \beta_{t}>0$. For this sequence and for some feasible allocation $\left(y_{t}, N_{t}, c_{t}, d_{t}, m_{t}\right)_{t \geq 0}$ with the same initial conditions, we define the objective function

$$
\liminf _{T \rightarrow+\infty} \sum_{t=0}^{T} \beta_{t}\left[U\left(c_{t}, d_{t+1}, m_{t}\right)-U\left(\bar{c}_{t}, \bar{d}_{t+1}, \bar{m}_{t}\right)\right] .
$$

If the maximum of the objective function on the set of $R C$-feasible allocations is reached in $\left(\bar{y}_{t}, \bar{N}_{t}, \bar{c}_{t}, \bar{d}_{t}, \bar{m}_{t}\right)_{t \geq 0}$, this allocation is $R C$-efficient. 
Remark 3 The assumption of the preceding lemma simply means that $\left(\bar{y}_{t}\right.$, $\left.\bar{N}_{t}, \bar{c}_{t}, \bar{d}_{t}, \bar{m}_{t}\right)_{t \geq 0}$ is weakly maximal in the sense of Brock (1970). Indeed, if $\left(\bar{y}_{t}, \bar{N}_{t}, \bar{c}_{t}, \bar{d}_{t}, \bar{m}_{t}\right)_{t \geq 0}$ maximizes (51), it cannot be overtaken by another feasible allocation.

Proof: Reductio ad absurdum. First, we can note that the objective function (51) is equal to zero for the allocation $\left(\bar{y}_{t}, \bar{N}_{t}, \bar{c}_{t}, \bar{d}_{t}, \bar{m}_{t}\right)_{t \geq 0}$. Let us assume that $\left(\bar{y}_{t}, \bar{N}_{t}, \bar{c}_{t}, \bar{d}_{t}, \bar{m}_{t}\right)_{t \geq 0}$ is a feasible RC-allocation that maximizes (51) in the set of feasible RC-allocations, and that this allocation is not RCefficient. In that case, it is RC-dominated by a feasible RC-allocation $\left(y_{t}\right.$, $\left.N_{t}, c_{t}, d_{t}, m_{t}\right)_{t \geq 0}$, such that

$$
\begin{aligned}
\forall t & \geq 0, U\left(c_{t}, d_{t+1}, m_{t}\right) \geq U\left(\bar{c}_{t}, \bar{d}_{t+1}, \bar{m}_{t}\right) \\
\exists t_{0} & \geq 0, \text { such that } U\left(c_{t_{0}}, d_{t_{0}+1}, m_{t_{0}}\right)>U\left(\bar{c}_{t_{0}}, \bar{d}_{t_{0}+1}, \bar{m}_{t_{0}}\right) .
\end{aligned}
$$

The consequence is that for each $T \geq t_{0}$

$$
\begin{aligned}
& \sum_{t=0}^{T} \beta_{t}\left[U\left(c_{t}, d_{t+1}, m_{t}\right)-U\left(\bar{c}_{t}, \bar{d}_{t+1}, \bar{m}_{t}\right)\right] \\
\geq & \beta_{t_{0}}\left[U\left(c_{t_{0}}, d_{t_{0}+1}, m_{t_{0}}\right)-U\left(\bar{c}_{t_{0}}, \bar{d}_{t_{0}+1}, \bar{m}_{t_{0}}\right)\right]>0 .
\end{aligned}
$$

The allocation $\left(\bar{y}_{t}, \bar{N}_{t}, \bar{c}_{t}, \bar{d}_{t}, \bar{m}_{t}\right)_{t \geq 0}$ does not maximize the objective function (51)

The preceding lemma provides a method for proving that a competitive equilibrium that converges in under-accumulation is RC-efficient: it is sufficient to prove that this allocation maximizes the objective function (51) in the set of feasible RC-allocations with an appropriate choice of weights $\left(\beta_{t}\right)$. This maximization problem is not relevant in the general case, however, because it is not concave. The lack of concavity is due to the presence of the term $m_{t} y_{t+1}$ in the resource constraint (38). Consequently, we only consider particular cases with CES utility and production functions, where the properties of (51) can be explicitly studied.

The optimization problem defined by lemma 2 can be solved in two stages: a static optimization problem that is concave in the general case; a dynamic optimization problem that is generally not concave. We begin by studying the static problem. This optimization problem is

$$
\left\{\begin{array}{c}
\max _{\left(c_{t}, d_{t+1}, m_{t}\right)} U\left(c_{t}, d_{t+1}, m_{t}\right) \\
\text { s. t. } m_{t} y_{t+1}=F\left(y_{t}-c_{t}-\phi m_{t}, m_{t}\right)-d_{t+1}
\end{array}\right.
$$

Our method consists of studying this problem in the particular case of CES utility and production functions before considering the dynamic problem. 
Even with these particular functions, however, we cannot find an explicit solution to this static problem. Below we introduce another optimization problem, which will be useful.

We define the following functions:

$$
\begin{aligned}
& v\left(y_{t}, y_{t+1}\right)=\left\{\begin{array}{c}
\max _{\left(c_{t}, d_{t+1}, m_{t}\right)} U\left(c_{t}, d_{t+1}, m_{t}\right) \\
\text { s. t. } m_{t} y_{t+1}=F\left(y_{t}-c_{t}-\phi m_{t}, m_{t}\right)-d_{t+1}
\end{array}\right. \\
& z\left(y_{t}, y_{t+1}\right)=\left\{\begin{array}{c}
\max _{\left(c_{t}, d_{t+1}, m_{t}, x_{t}\right)} U\left(c_{t}, d_{t+1}, m_{t}\right) \\
\text { s. t. } x_{t} y_{t+1}=F\left(y_{t}-c_{t}-\phi m_{t}, x_{t}\right)-d_{t+1}
\end{array}\right.
\end{aligned}
$$

By definition, we have $v\left(y_{t}, y_{t+1}\right) \leq z\left(y_{t}, y_{t+1}\right)$. Indeed, the first program looks like the second program with an additional constraint: $x_{t}=m_{t}$.

Recalling that $\bar{y}_{t}=\bar{w}_{t}$ along an intertemporal equilibrium, the objective function defined by (51) can now be set as

$$
\liminf _{T \rightarrow+\infty} \sum_{t=0}^{T} \beta_{t}\left[v\left(y_{t}, y_{t+1}\right)-v\left(\bar{w}_{t}, \bar{w}_{t+1}\right)\right],
$$

and it is also possible to define a second objective as

$$
\liminf _{T \rightarrow+\infty} \sum_{t=0}^{T} \beta_{t}\left[z\left(y_{t}, y_{t+1}\right)-z\left(\bar{w}_{t}, \bar{w}_{t+1}\right)\right] .
$$

The following lemma establishes two properties that are satisfied by $v$ and $z$. First, these two functions coincide when $\left(y_{t}, y_{t+1}\right)$ is taken along a competitive trajectory: $\left(y_{t}, y_{t+1}\right)=\left(\bar{w}_{t}, \bar{w}_{t+1}\right)$. Secondly, for an appropriate choice of the sequence $\left(\beta_{t}\right)$, the competitive equilibrium satisfies the firstorder conditions of the two dynamic optimization problems defined by (59) and (60).

Lemma 3 Let $\left(\bar{y}_{t}, \bar{N}_{t}, \bar{c}_{t}, \bar{d}_{t}, \bar{m}_{t}\right)_{t>0}$ be a competitive equilibrium with $\bar{w}_{t}=$ $\bar{y}_{t}$ as the competitive wage and $\bar{R}_{t+1}=F_{K}^{\prime}\left(\bar{y}_{t}-\bar{c}_{t}-\phi \bar{m}_{t}, \bar{m}_{t}\right)$ as the competitive interest factor. We have:

1. $v\left(\bar{w}_{t}, \bar{w}_{t+1}\right)=z\left(\bar{w}_{t}, \bar{w}_{t+1}\right)=U\left(\bar{c}_{t}, \bar{d}_{t+1}, \bar{m}_{t}\right)$.

2. Let $\left(\beta_{t}\right)$ be the sequence defined by: $\beta_{0}=1$ and

$$
\forall t \geq 0, \frac{\beta_{t+1}}{\beta_{t}}=\frac{U_{c}^{\prime}\left(\bar{c}_{t}, \bar{d}_{t+1}, \bar{m}_{t}\right) \bar{m}_{t}}{U_{c}^{\prime}\left(\bar{c}_{t+1}, \bar{d}_{t+2}, \bar{m}_{t+1}\right) \bar{R}_{t+1}},
$$


we have $\forall t \geq 0$

$$
\begin{aligned}
& \beta_{t} v_{2}^{\prime}\left(\bar{w}_{t}, \bar{w}_{t+1}\right)+\beta_{t+1} v_{1}^{\prime}\left(\bar{w}_{t+1}, \bar{w}_{t+2}\right)=0 \\
& \beta_{t} z_{2}^{\prime}\left(\bar{w}_{t}, \bar{w}_{t+1}\right)+\beta_{t+1} z_{1}^{\prime}\left(\bar{w}_{t+1}, \bar{w}_{t+2}\right)=0 .
\end{aligned}
$$

Proof: see Appendix 2 .

The first result is obtained by showing that the first order conditions of the two static optimization programs coincide when $\left(y_{t}, y_{t+1}\right)=\left(\bar{w}_{t}, \bar{w}_{t+1}\right)$. The second result proves that the marginal conditions of the two dynamic optimization programs (59) and (60) are satisfied by $y_{t}=\bar{w}_{t} \forall t$, when $\beta_{t}$ is defined by (61).

We can now consider the trajectory of the competitive equilibrium $\left(\bar{y}_{t}, \bar{N}_{t}\right.$, $\left.\bar{c}_{t}, \bar{d}_{t}, \bar{m}_{t}\right)_{t \geq 0}$ obtained using the CES production technology given by (13), or by (14) in the limited case of an elasticity of substitution equal to one, and a CES utility function given by (1). We know that the steady state $\bar{k}$ is reached from period 1 , and that it is unique under the assumption $\tau+\rho \geq 1$. Denoting the stationary values of the interest factor and the fertility level by $\bar{R}=R(\bar{k})$ and $\bar{m}$, respectively, we obtain the following result:

Proposition 3 Assume that $\tau+\rho \geq 1$ (the competitive equilibrium is determined), that $\tau \leq 1$ (production factors are complements in production) and that the competitive economy converges in under-accumulation $(\bar{R}>\bar{m})$. Then, if $\tau+\rho \leq 2$, the competitive equilibrium is $R C$-efficient.

Proof: see Appendix 3

The result is obtained by explicitly calculating the function $z\left(y_{t}, y_{t+1}\right)$ and proving that the objective function

$$
\liminf _{T \rightarrow+\infty} \sum_{t=0}^{T} \beta_{t}\left[z\left(y_{t}, y_{t+1}\right)-z\left(\bar{w}_{t}, \bar{w}_{t+1}\right)\right]
$$

reaches a global maximum in $y_{t}=\bar{w}_{t}$. As $v\left(y_{t}, y_{t+1}\right) \leq z\left(y_{t}, y_{t+1}\right)$ with $v\left(\bar{w}_{t}, \bar{w}_{t+1}\right)=z\left(\bar{w}_{t}, \bar{w}_{t+1}\right)$, the objective function

$$
\liminf _{T \rightarrow+\infty} \sum_{t=0}^{T} \beta_{t}\left[v\left(y_{t}, y_{t+1}\right)-v\left(\bar{w}_{t}, \bar{w}_{t+1}\right)\right]
$$

reaches a global maximum in $y_{t}=\bar{w}_{t}$.

The intuition behind Proposition 3 is that an allocation can Paretoimprove a competitive equilibrium converging in under-accumulation, if it 
leads, in the long run, to a fertility level higher than the interest factor. Our result gives two arguments capable of precluding this increase in the fertility level. The first argument is the complementarity of production factors. Indeed, the increase in fertility is linked to a fall in capital accumulation. If capital and labor are substitutable, this change is not too detrimental to production; if capital and labor are complementary, however, it can lead to a significant drop in resources. The second argument is the complementarity of consumption and fertility in the utility function. If $c, d$ and $m$ are highly substitutable, the increase in $m$ allows for a substantial reduction in $c$ and $d$, which is compatible with a constant level of utility. When they are complementary, however, the admissible fall of $c$ and $d$ is low.

Proposition 3 shows that a trade-off between the two substitution elasticities in production and utility exists, such that the competitive equilibrium (converging in under-accumulation) is RC-efficient. This condition is the same as the one found in Michel and Pestieau (1993), who are interested in the optimal fertility rate in a framework of exogenous fertility. In their model, the condition guarantees that the serendipity theorem holds.

Michel and Wigniolle (2006) provide a general condition which ensures Pareto-efficiency for the competitive equilibrium of an economy endowed with general utility and production functions: an intertemporal equilibrium converging toward a stationary state such that $\phi \bar{R}>\bar{w}$ is RC-efficient. This condition is stronger than under-accumulation. Indeed, at the steady state of the competitive economy, we have: $\phi \bar{m} \leq \bar{w}<\phi \bar{R} \Rightarrow \bar{m}<\bar{R}$.

With respect to this general condition, our results in Proposition 3 are more precise for an economy endowed with a CES utility and production functions. We have proved that if $\rho+\tau \geq 1, \tau \leq 1$ and $\rho+\tau \leq 2$ hold, underaccumulation is sufficient for RC-efficiency of the competitive equilibrium. The condition $\phi \bar{R}>\bar{w}$ is stronger and not relevant in this case.

Proposition 3 gives sufficient conditions for RC-efficiency of a competitive equilibrium in under-accumulation. But it is unlikely that these conditions are necessary. Proposition 2 allows us to conclude on the inefficiency only for high values of $\tau$. There exists a large range of the parameters $(\tau, \rho)$ for which no conclusion is reached. Indeed, even if $\tau$ and $\rho$ do not satisfy the conditions given in Proposition 3, building a RC-allocation that dominates the competitive equilibrium needs extra assumptions on other parameters. For instance, in Proposition 2, we also need some constraints on the parameters $A$ and $b$ of the production technology, on the child cost $\phi$, and on the coefficients $\beta$ and $\gamma$ of the utility function.

Our results give some insights on the role of the two parameters $\tau$ and $\rho$, but the understanding is not complete and some further results could be achieved. More precisely, the proofs of Propositions 2 and 3 give some 
intuitions that we were not able to prove. In Proposition 2, the assumption that $\tau$ is sufficiently high plays a crucial role. A value of $\tau$ greater than one implies that the marginal productivity of capital tends to a finite value when the capital stock tends toward 0. It is also well known that this case allows the existence of unbounded realizable trajectories for the variable $k_{t}$. These properties allow us to build an allocation that $\mathrm{RC}$-dominates the competitive equilibrium in under-accumulation. Now, assuming $\tau \leq 1$ and high enough $\rho$, such that the assumption $\rho+\tau \leq 2$ in Proposition 3 is violated, or even such that $\rho \rightarrow+\infty$, is it possible to construct such an allocation dominating the competitive equilibrium? We have not succeeded in answering this question. Conversely, it could be true that the condition $\tau \leq 1$ alone, without the extra condition $\rho+\tau \leq 2$, is sufficient to ensure RC-efficiency, but we have not succeeded in proving this property.

\section{Conclusion}

This article has studied the efficiency of the competitive equilibrium for an overlapping generations economy with endogenous fertility. Utility and production functions were assumed to be CES. We have given a sufficient condition, which ensures the existence and uniqueness of a competitive equilibrium: the sum of the two elasticities must not be smaller than one. Then, we have exhibited a set of parameters such that a competitive equilibrium in under-accumulation is not efficient. Finally, we have proved that a competitive equilibrium converging in under-accumulation is Pareto-efficient if the sum of the two elasticities of substitution is not greater than two.

It would be interesting to study whether these results remain true in considering more general environments. Notably, a generalization could emerge concerning the cost of children. In our simple framework, this cost is constant for each child. Conde-Ruiz, Giménez and Perez-Nievas (2004) consider a more general formulation where the cost is a convex function of the number of children. Another interesting generalization could be to introduce a cost in time. Under this assumption, children have an opportunity cost that depends on the wage. According to Greenwood, Seshadri and Vandenbroucke (2005), the rise of this opportunity cost has played a crucial role in the historical decline in fertility observed in America over the last 200 years.

Using policy-oriented models with endogenous fertility and applying efficiency criteria to these models could be an important topic. Indeed, efficiency criteria should influence the design of economic policies such as PAYG pension systems or child benefits, as appears from Conde-Ruiz, Giménez and Perez-Nievas' results (2004). 


\section{Appendix 1: proof of Proposition 2.}

We introduce the following notation in what follows

$$
\gamma_{i}[R(\bar{k})]=\bar{\gamma}_{i}
$$

We assume that the competitive equilibrium is in under-accumulation. This property is expressed by the condition

$$
\bar{R}>\bar{m}=\frac{\bar{\gamma}_{3} \bar{w}}{\phi}
$$

or

$$
\bar{\gamma}_{3}<\frac{\phi \bar{R}}{\bar{w}}
$$

We can then build a feasible RC-allocation starting from the stationary state $k_{0}=\bar{k}$ and $m_{-1}=\bar{m}$, such that: $\forall t \geq 0, c_{t}=\bar{c}, d_{t}=\bar{d}, m_{t}=m$, and $k_{t}$ satisfies the resource constraint

$$
m k_{t+1}=f\left(k_{t}\right)-\bar{c}-\frac{\bar{d}}{m_{t-1}}-\phi m .
$$

In $t=0$, as $m_{-1}$ is given and equal to $\bar{m}$, this condition is

$$
m k_{1}=f(\bar{k})-\bar{c}-\frac{\bar{d}}{\bar{m}}-\phi m .
$$

As $\bar{d}=\bar{R} \bar{s}=\bar{R} \bar{m} \bar{k}$, the term $f(\bar{k})-\bar{d} / \bar{m}$ is equal to $f(\bar{k})-\overline{R k}=\bar{w}$. The preceding condition becomes

$$
m k_{1}=\bar{w}-\bar{\gamma}_{1} \bar{w}-\phi m
$$

For $t \geq 1,(69)$ becomes

$$
m k_{t+1}=f\left(k_{t}\right)-\bar{c}-\frac{\bar{d}}{m}-\phi m .
$$

We set $\mu^{2} \equiv \bar{d} / \phi=\bar{\gamma}_{2} \bar{R} \bar{w} / \phi$. We can write

$$
m k_{t+1}=f\left(k_{t}\right)-\bar{\gamma}_{1} \bar{w}-\phi\left(\frac{\mu^{2}}{m}+m\right) .
$$

We choose $m=\mu$, which is the value that minimizes the last term of the equation. At each date $t$, the condition $k_{t}>0$ must hold. As

$$
f(k)>w(k)=A b\left(k^{\frac{\tau-1}{\tau}}+b\right)^{\frac{1}{\tau-1}}>A b^{\frac{\tau}{\tau-1}},
$$


we obtain sufficient conditions that respectively ensure that $k_{1}>0$ and $k_{t+1}>0 \forall t \geq 1$,

$$
\begin{aligned}
A b^{\frac{\tau}{\tau-1}}-\bar{\gamma}_{1} \bar{w}-\phi \mu & >0 \\
A b^{\frac{\tau}{\tau-1}}-\bar{\gamma}_{1} \bar{w}-2 \phi \mu & >0
\end{aligned}
$$

The only condition that must hold is

$$
A b^{\frac{\tau}{\tau-1}}>\bar{\gamma}_{1} \bar{w}+2 \phi \mu
$$

Finally, we impose the inequality $\mu>\bar{m}$, which leads to

$$
\phi \bar{R} / \bar{w}>\bar{\gamma}_{3}^{2} / \bar{\gamma}_{2}
$$

Condition (78) implies $U(\bar{c}, \bar{d}, \mu)>U(\bar{c}, \bar{d}, \bar{m})$ as $\mu>\bar{m}$. All utilities are higher along the feasible trajectory with $m_{t}=\mu$.

The three conditions (68), (77) and (78) remain to be studied at the limit when $\tau \rightarrow+\infty$. When $\tau \rightarrow+\infty, \lim \bar{R}=A$ and $\lim \bar{w}=A b$.

Conditions (68) and (78) are equivalent to

$$
\phi / b>\max \left[\gamma_{2}(A), \gamma_{3}^{2}(A) / \gamma_{2}(A)\right] .
$$

Condition (77) can be written as

$$
2 \phi \mu<A b\left[1-\gamma_{1}(A)\right]=A b\left[\gamma_{2}(A)+\gamma_{3}(A)\right] .
$$

Taking the square of the preceding inequality, we obtain

$$
4 \phi^{2} \mu^{2}=4 \phi \gamma_{2}(A) A^{2} b<A^{2} b^{2}\left[\gamma_{2}(A)+\gamma_{3}(A)\right]^{2},
$$

or

$$
\phi / b<\frac{\left[\gamma_{2}(A)+\gamma_{3}(A)\right]^{2}}{4 \gamma_{2}(A)} .
$$

By (79) and (82), we must have

$$
\frac{\gamma_{3}^{2}(A)}{\gamma_{2}(A)}<\frac{\left[\gamma_{2}(A)+\gamma_{3}(A)\right]^{2}}{4 \gamma_{2}(A)}
$$

which implies

$$
\gamma_{2}(A)>\gamma_{3}(A)
$$

And, if $\gamma_{2}(A)>\gamma_{3}(A)$, then $\max \left[\gamma_{2}(A), \gamma_{3}^{2}(A) / \gamma_{2}(A)\right]=\gamma_{2}(A)$. 
Finally, we have proved that the conditions that are to be satisfied are

$$
\begin{aligned}
& \gamma_{3}(A)<\gamma_{2}(A), \\
& \gamma_{3}(A)<\phi / b<\frac{\left[\gamma_{2}(A)+\gamma_{3}(A)\right]^{2}}{4 \gamma_{2}(A)},
\end{aligned}
$$

where the inequality $\gamma_{3}(A)<\frac{\left[\gamma_{2}(A)+\gamma_{3}(A)\right]^{2}}{4 \gamma_{2}(A)}$ is a consequence of $\gamma_{3}(A)<\gamma_{2}(A)$.

Using (11) and (12), (85) gives

$$
\gamma^{\rho} \phi^{1-\rho} \beta^{-\rho} A^{1-\rho}<1
$$

that can be written

$$
\rho\left[\ln \left(\frac{\gamma}{\beta}\right)-\ln (A \phi)\right]+\ln (A \phi)<0 .
$$

As $\rho>0$, it is straightforward to prove that if one condition among (47) (48) or (49) is satisfied, then $\gamma_{2}(A)>\gamma_{3}(A)$. Moreover, we know that in this case $\gamma_{3}(A)<\frac{\left[\gamma_{2}(A)+\gamma_{3}(A)\right]^{2}}{4 \gamma_{2}(A)}$.

Considering now (86), it can be interpreted as a condition on $b$ that can be written

$$
\frac{4 \gamma_{2}(A) \phi}{\left[\gamma_{2}(A)+\gamma_{3}(A)\right]^{2}}<b<\frac{\phi}{\gamma_{3}(A)} \text {. }
$$

Using (11) and (12), this last condition becomes (50).

Finally, when conditions given in Proposition 2 hold, conditions (68), (77) and (78) are satisfied in the limit case when $\tau \rightarrow+\infty$. By continuity, they are satisfied when $\tau$ is large enough.

As a last comment, we see that the inequality $\gamma_{3}(A)<\gamma_{2}(A)$ implies $\gamma_{3}(A)<\frac{\left[\gamma_{2}(A)+\gamma_{3}(A)\right]^{2}}{4 \gamma_{2}(A)}<\gamma_{2}(A)$. When (85) and (86) hold, we have

$$
\phi / b<\gamma_{2}(A)
$$

which is equivalent to the inequality

$$
\mu>\bar{R}
$$

when $\tau \rightarrow+\infty$. Consequently, the allocation $(\bar{c}, \bar{d}, \mu)$ that has been built is associated with over-accumulation.

\section{Appendix 2: proof of lemma 3.}

The first part of the lemma is obtained by considering the two static optimization programs, which are both concave. 
The first order conditions for the first program are

$$
U_{c_{t}}^{\prime}=U_{d_{t+1}}^{\prime}=U_{m_{t}}^{\prime} \frac{F_{K_{t+1}}^{\prime}}{\phi F_{K_{t+1}}^{\prime}-F_{L_{t+1}}^{\prime}+y_{t+1}} .
$$

For $\left(y_{t}, y_{t+1}\right)=\left(\bar{w}_{t}, \bar{w}_{t+1}\right)$ and $\left(c_{t}, d_{t+1}, m_{t}\right)=\left(\bar{c}_{t}, \bar{d}_{t+1}, \bar{m}_{t}\right)$, these conditions are satisfied, as they correspond to the conditions of the consumer maximization program along the competitive equilibrium. The resource constraint is identical to the budget constraint of the consumer.

The first order conditions for the second program are

$$
\begin{aligned}
U_{c_{t}}^{\prime} & =U_{d_{t+1}}^{\prime}=\frac{U_{m_{t}}^{\prime}}{\phi}, \\
F_{L_{t+1}}^{\prime} & =y_{t+1} .
\end{aligned}
$$

All these conditions are satisfied when $\left(y_{t}, y_{t+1}\right)=\left(\bar{w}_{t}, \bar{w}_{t+1}\right)$ and $\left(c_{t}, d_{t+1}, m_{t}\right)=$ $\left(\bar{c}_{t}, \bar{d}_{t+1}, \bar{m}_{t}\right)$.

These two optimization programs also make it possible to find the following properties

$$
\begin{aligned}
& v_{1}^{\prime}\left(y_{t}, y_{t+1}\right)=U_{c_{t}}^{\prime} \text { and } v_{2}^{\prime}\left(y_{t}, y_{t+1}\right)=-U_{c_{t}}^{\prime} \frac{m_{t}}{F_{K_{t+1}}^{\prime}} \\
& z_{1}^{\prime}\left(y_{t}, y_{t+1}\right)=U_{c_{t}}^{\prime} \text { and } z_{2}^{\prime}\left(y_{t}, y_{t+1}\right)=-U_{c_{t}}^{\prime} \frac{x_{t}}{F_{K_{t+1}}^{\prime}} .
\end{aligned}
$$

The second result is obtained by using the following properties

$$
\begin{aligned}
& v_{1}^{\prime}\left(\bar{w}_{t+1}, \bar{w}_{t+2}\right)=U_{c_{t+1}}^{\prime}\left(\bar{c}_{t+1}, \bar{d}_{t+2}, \bar{m}_{t+1}\right) \text { and } v_{2}^{\prime}\left(\bar{w}_{t}, \bar{w}_{t+1}\right)=-\frac{U_{c_{t}}^{\prime}\left(\bar{c}_{t}, \bar{d}_{t+1}, \bar{m}_{t}\right) \bar{m}_{t}}{F_{K}^{\prime}\left(\bar{K}_{t+1}, \bar{N}_{t+1}\right)} \\
& z_{1}^{\prime}\left(\bar{w}_{t+1}, \bar{w}_{t+2}\right)=U_{c_{t+1}}^{\prime}\left(\bar{c}_{t+1}, \bar{d}_{t+2}, \bar{m}_{t+1}\right) \text { and } z_{2}^{\prime}\left(\bar{w}_{t}, \bar{w}_{t+1}\right)=-\frac{U_{c_{t}}^{\prime}\left(\bar{c}_{t}, \bar{s}_{t+1}, \bar{m}_{t}\right) \bar{m}_{t}}{F_{K}^{\prime}\left(\bar{K}_{t+1}, \bar{N}_{t+1}\right)} .
\end{aligned}
$$

From the definition of the sequence $\beta_{t}$, the result is proved.

\section{Appendix 3: proof of Proposition 3.}

We separately consider the two cases: $\tau<1$ and $\tau=1$.

First case: $\tau<1$.

For CES production and utility functions, we can calculate the explicit form of the function $z$. We can consider the utility function

$$
\ln \left(c_{t}^{\frac{\rho-1}{\rho}}+\beta d_{t+1}^{\frac{\rho-1}{\rho}}+\gamma m_{t}^{\frac{\rho-1}{\rho}}\right)^{\frac{\rho}{\rho-1}} .
$$

$z$ is defined by 


$$
\begin{gathered}
\max _{\left(c_{t}, d_{t+1}, m_{t}, x_{t}\right)} \ln \left(c_{t}^{\frac{\rho-1}{\rho}}+\beta d_{t+1}^{\frac{\rho-1}{\rho}}+\gamma m_{t}^{\frac{\rho-1}{\rho}}\right)^{\frac{\rho}{\rho-1}} \\
\text { s. t. } x_{t} y_{t+1}=A\left[\left(y_{t}-c_{t}-\phi m_{t}\right)^{\frac{\tau-1}{\tau}}+b\left(x_{t}\right)^{\frac{\tau-1}{\tau}}\right]^{\frac{\tau}{\tau-1}}-d_{t+1}
\end{gathered}
$$

First, we independently consider the maximization with respect to $x_{t}$ (as $x_{t}$ only appears in the constraint and not in the objective)

$$
\max _{x_{t}} d_{t+1}=A\left[\left(y_{t}-c_{t}-\phi m_{t}\right)^{\frac{\tau-1}{\tau}}+b\left(x_{t}\right)^{\frac{\tau-1}{\tau}}\right]^{\frac{\tau}{\tau-1}}-x_{t} y_{t+1}
$$

Before solving this program, we note that, as $\tau<1$,

$$
A\left[\left(y_{t}-c_{t}-\phi m_{t}\right)^{\frac{\tau-1}{\tau}}+b\left(x_{t}\right)^{\frac{\tau-1}{\tau}}\right]^{\frac{\tau}{\tau-1}}<A b^{\frac{\tau}{\tau-1}} x_{t} .
$$

We obtain:

$$
d_{t+1}<\left(A b^{\frac{\tau}{\tau-1}}-y_{t+1}\right) x_{t}
$$

As $d_{t+1}$ must be positive, however, the condition $y_{t+1}<A b^{\frac{\tau}{\tau-1}}$ must be satisfied along any feasible trajectory.

When $y_{t+1}<A b^{\frac{\tau}{\tau-1}}$, the program (100) has an interior solution. We find

$$
b\left(x_{t}\right)^{\frac{\tau-1}{\tau}}=\frac{\left(y_{t}-c_{t}-\phi m_{t}\right)^{\frac{\tau-1}{\tau}}}{\left(\frac{y_{t+1}}{A b^{\frac{\tau}{\tau-1}}}\right)^{\tau-1}-1} .
$$

Replacing $b\left(x_{t}\right)^{\frac{\tau-1}{\tau}}$ in the constraint, we obtain

$$
d_{t+1}=A\left(y_{t}-c_{t}-\phi m_{t}\right)\left[1-\left(\frac{y_{t+1}}{A b^{\frac{\tau}{\tau-1}}}\right)^{1-\tau}\right]^{\frac{1}{1-\tau}}
$$

so that we can also write

$$
c_{t}+\phi m_{t}+h\left(y_{t+1}\right) d_{t+1}=y_{t},
$$

with

$$
h\left(y_{t+1}\right)=b^{\frac{\tau}{\tau-1}}\left[\left(A b^{\frac{\tau}{\tau-1}}\right)^{1-\tau}-\left(y_{t+1}\right)^{1-\tau}\right]^{\frac{1}{\tau-1}} .
$$

It will be useful to keep in mind the following property of the function $h$

$$
\frac{h^{\prime}\left(y_{t+1}\right) y_{t+1}}{h\left(y_{t+1}\right)}=\frac{\left(y_{t+1}\right)^{1-\tau}}{\left(A b^{\frac{\tau}{\tau-1}}\right)^{1-\tau}-\left(y_{t+1}\right)^{1-\tau}} .
$$


It is straightforward to check that the function $1 / h(y)$ is the factor price frontier that gives the interest factor $R$ with respect to the wage $y$ when firms' behavior is competitive.

Secondly, we must consider the program

$$
\begin{gathered}
\max _{\left(c_{t}, d_{t+1}, m_{t}\right)} \ln \left(c_{t}^{\frac{\rho-1}{\rho}}+\beta d_{t+1}^{\frac{\rho-1}{\rho}}+\gamma m_{t}^{\frac{\rho-1}{\rho}}\right)^{\frac{\rho}{\rho-1}} \\
\text { s. t. } c_{t}+\phi m_{t}+h\left(y_{t+1}\right) d_{t+1}=y_{t}
\end{gathered}
$$

The indirect utility associated with this program is by definition the function $z$

$$
z\left(y_{t}, y_{t+1}\right)=\ln y_{t}+\frac{1}{\rho-1} \ln \left[1+\gamma^{\rho} \phi^{1-\rho}+\beta^{\rho} h\left(y_{t+1}\right)^{1-\rho}\right],
$$

which is additively separable as the sum of a function of $y_{t}$ and a function of $y_{t+1}$.

Let us now consider the objective

$$
\liminf _{T \rightarrow+\infty} \sum_{t=0}^{T} \beta_{t}\left[z\left(y_{t}, y_{t+1}\right)-v\left(\bar{w}_{t}, \bar{w}_{t+1}\right)\right],
$$

with the sequence $\left(\beta_{t}\right)$ defined by (61). For a CES utility function, we know from Section 3.3 that $\bar{w}_{t}$ is constant: $\forall t \geq 1, \bar{w}_{t}=\bar{w}$. As the function $z$ is additively separable, it is possible to match together the two terms that depend on $y_{t}(\forall t \geq 1)$

$$
Z\left(y_{t}\right) \equiv \frac{\beta_{t-1}}{\rho-1} \ln \left[1+\gamma^{\rho} \phi^{1-\rho}+\beta^{\rho} h\left(y_{t}\right)^{1-\rho}\right]+\beta_{t} \ln y_{t}
$$

We study this function on $\left(0, A b^{\frac{\tau}{\tau-1}}\right)$.

From the lemma 3, we know that the derivative of this function cancels out when $y_{t}=\bar{w}_{t}=\bar{w}$ for $t \geq 1$. This derivative is calculated in using (107)

$$
Z^{\prime}\left(y_{t}\right)=\frac{\beta_{t-1}}{y_{t}}\left\{\frac{\beta_{t}}{\beta_{t-1}}-\frac{\beta^{\rho} h\left(y_{t}\right)^{1-\rho}\left[\frac{\left(y_{t}\right)^{1-\tau}}{\left(A b^{\frac{\tau}{\tau-1}}\right)^{1-\tau}-\left(y_{t}\right)^{1-\tau}}\right]}{1+\gamma^{\rho} \phi^{1-\rho}+\beta^{\rho} h\left(y_{t}\right)^{1-\rho}}\right\} .
$$

First, we prove that the function $Z$ has a global maximum on $\left(0, A b^{\frac{\tau}{\tau-1}}\right)$ when $y_{t}=\bar{w}_{t}=\bar{w}$. 
We establish this property in introducing the function

$$
Y\left(y_{t}\right)=\frac{h\left(y_{t}\right)^{1-\rho}\left[\frac{\left(y_{t}\right)^{1-\tau}}{\left(A b^{\frac{\tau}{\tau-1}}\right)^{1-\tau}-\left(y_{t}\right)^{1-\tau}}\right]}{1+\gamma^{\rho} \phi^{1-\rho}+\beta^{\rho} h\left(y_{t}\right)^{1-\rho}},
$$

which is such that

$$
Z^{\prime}\left(y_{t}\right)=\frac{\beta_{t-1}}{y_{t}}\left[\frac{\beta_{t}}{\beta_{t-1}}-\beta^{\rho} Y\left(y_{t}\right)\right] .
$$

If the function $Y\left(y_{t}\right)$ is strictly increasing, as $\beta_{t} / \beta_{t-1}-\beta^{\rho} Y\left(y_{t}\right)$ cancels out when $y_{t}=\bar{w}_{t}=\bar{w}$, we can deduce that $Z^{\prime}\left(y_{t}\right)<0$ for $y_{t}<\bar{w}$ and that $Z^{\prime}\left(y_{t}\right)>0$ for $y_{t}>\bar{w} \cdot y_{t}=\bar{w}$ is the global maximum of $Z$ on $\left(0, A b^{\frac{\tau}{\tau-1}}\right)$.

To prove that $Y\left(y_{t}\right)$ is strictly increasing, we calculate

$$
\frac{Y^{\prime}(y)}{Y(y)}=(1-\rho) \frac{h^{\prime}(y)}{h(y)}+\frac{(1-\tau)}{y}+(1-\tau) \frac{y^{-\tau}}{\left(A b^{\frac{\tau}{\tau-1}}\right)^{1-\tau}-y^{1-\tau}}-\frac{(1-\rho) \beta^{\rho} h^{\prime}(y) h(y)^{-\rho}}{1+\gamma^{\rho} \phi^{1-\rho}+\beta^{\rho} h(y)^{1-\rho}}
$$

Using (107), we have

$$
\frac{Y^{\prime}(y)}{Y(y)}=\frac{\left(1+\gamma^{\rho} \phi^{1-\rho}\right)\left[(1-\rho) y^{1-\tau}+(1-\tau)\left(A b^{\frac{\tau}{\tau-1}}\right)^{1-\tau}\right]+\beta^{\rho} h(y)^{1-\rho}(1-\tau)\left(A b^{\frac{\tau}{\tau-1}}\right)^{1-\tau}}{y\left[\left(A b^{\frac{\tau}{\tau-1}}\right)^{1-\tau}-y^{1-\tau}\right]\left[1+\gamma^{\rho} \phi^{1-\rho}+\beta^{\rho} h(y)^{1-\rho}\right]} .
$$

By assumption, as $\tau<1$, the term $\beta^{\rho} h(y)^{1-\rho}(1-\tau)\left(A b^{\frac{\tau}{\tau-1}}\right)^{1-\tau}$ is positive.

If $\rho<1$, as $\tau<1$ the other term is positive and $Y^{\prime}(y)>0$.

If $\rho>1$, as $y<A b^{\frac{\tau}{\tau-1}}$ and $\rho>1$, we obtain

$$
(1-\rho) y^{1-\tau}+(1-\tau)\left(A b^{\frac{\tau}{\tau-1}}\right)^{1-\tau}>\left(A b^{\frac{\tau}{\tau-1}}\right)^{1-\tau}(1-\rho+1-\tau) .
$$

A sufficient condition for obtaining $Y^{\prime}(y) / Y(y)>0$ is $2-\rho-\tau \geq 0$. Finally, we have proved that if

$$
\rho+\tau \leq 2,
$$

the function $Z$ has a global maximum on $\left(0, A b^{\frac{\tau}{\tau-1}}\right)$ when $y_{t}=\bar{w}_{t}=\bar{w}$.

Next, we must prove that the objective function is maximum at this point. This result is achieved by writing 


$$
\begin{gathered}
D_{T} \equiv \sum_{t=0}^{T} \beta_{t}\left[z\left(y_{t}, y_{t+1}\right)-v\left(\bar{w}_{t}, \bar{w}_{t+1}\right)\right]= \\
\sum_{t=0}^{T} \beta_{t}\left[Z\left(y_{t}\right)-Z(\bar{w})\right]+ \\
\frac{\beta_{T}}{\rho-1}\left\{\ln \left[1+\gamma^{\rho} \phi^{1-\rho}+\beta^{\rho} h\left(y_{T+1}\right)^{1-\rho}\right]-\ln \left[1+\gamma^{\rho} \phi^{1-\rho}+\beta^{\rho} h(\bar{w})^{1-\rho}\right]\right\} .
\end{gathered}
$$

As $Z(y)$ reaches a global maximum for $y=\bar{w}$, the first term is negative

$$
\sum_{t=0}^{T} \beta_{t}\left[Z\left(y_{t}\right)-Z(\bar{w})\right] \leq 0 .
$$

As $h(y)$ is an increasing function, the second term is a decreasing function of $y_{T+1}$. This term is smaller than:

$$
\frac{\beta_{T}}{\rho-1}\left\{\ln \left[1+\gamma^{\rho} \phi^{1-\rho}+\beta^{\rho} h(0)^{1-\rho}\right]-\ln \left[1+\gamma^{\rho} \phi^{1-\rho}+\beta^{\rho} h(\bar{w})^{1-\rho}\right]\right\} \equiv E_{T} .
$$

Finally, we obtain

$$
D_{T} \leq E_{T},
$$

with

$$
\lim _{T \rightarrow+\infty} E_{T}=0
$$

since $\lim _{T \rightarrow+\infty} \beta_{T}=0$.

We can conclude that

$$
\lim _{T \rightarrow+\infty} D_{T} \leq 0
$$

Second case: $\tau=1$.

In this case, the production function is a Cobb-Douglas given by (14). We use the same method as for $\tau<1$. It is easy to check that equation (109) remains true with a function $h(y)$ defined by

$$
h(y)=\frac{y^{\frac{1-\alpha}{\alpha}}}{\alpha A^{\frac{1}{1-\alpha}}(1-\alpha)^{\frac{1-\alpha}{\alpha}}} .
$$

Equation (114) remains identical, with a function $Y(y)$ defined by

$$
Y(y)=\frac{\frac{1-\alpha}{\alpha} h(y)^{1-\rho}}{1+\gamma^{\rho} \phi^{1-\rho}+\beta^{\rho} h(y)^{1-\rho}}=\frac{\frac{1-\alpha}{\alpha}}{\left(1+\gamma^{\rho} \phi^{1-\rho}\right) h(y)^{\rho-1}+\beta^{\rho}} .
$$


If $\rho<1$, as $h(y)$ is a (strictly) increasing function, $Y(y)$ is (strictly) increasing, and the property is proved.

When $\rho=1, Y(y)$ is constant. As we know that $Z^{\prime}(\bar{w})=0$, we obtain that $Z^{\prime}(y)=0 \forall y$ and finally that $Z(y)$ is constant. Using the same reasoning as for $\tau<1, \liminf _{T \rightarrow+\infty} \sum_{t=0}^{T} \beta_{t}\left[z\left(y_{t}, y_{t+1}\right)-v\left(\bar{w}_{t}, \bar{w}_{t+1}\right)\right]=0$. For $y_{t}=$ $\bar{w}_{t}, \liminf _{T \rightarrow+\infty} \sum_{t=0}^{T} \beta_{t}\left[v\left(y_{t}, y_{t+1}\right)-v\left(\bar{w}_{t}, \bar{w}_{t+1}\right)\right]$ reaches its maximum value and the property is proved. 


\section{References}

Becker, G. S. (1960). An economic analysis of fertility. In Demographic and Economic Change in Developed Countries. Universities-National Bureau Conference Series, no. 11. Princeton Univ. Press.

Birdsall, N. (1988). Economic approaches to population growth. In Chenery, H. and Srinivasan, T. N. (eds) Handbook of Development Economics. Amsterdam New York Oxford: North-Holland, Vol. I, 477542.

Brock, W. A. (1970). On the existence of weakly-maximal programmes in a multi-sector economy. Review of Economic Studies 37: 275-280.

Conde-Ruiz, J.I., Gimenez, E.L. and Perez-Nievas, M. (2004). Millian efficiency with endogenous fertility. Documento de Trabajo 2004-13. FEDEA, Madrid.

De la Croix, D. and Michel, P. (2002). A Theory of Economic Growth: Dynamics and Policy in Overlapping Generations. Cambridge Univ. Press.

Diamond, P. (1965). National debt in a neoclassical growth model. American Economic Review 55: 1126-1150.

Eckstein, Z. and Wolpin, K. (1985). Endogenous fertility and optimal population size. Journal of Public Economics 27: 93-106.

Golosov, M., Jones, L.E. and Tertilt, M. (2007). Efficiency with endogenous population growth. Econometrica 75(4): 1039-1071.

Michel, P. and Pestieau, P. (1993). Population growth and optimality: when does serendipity hold. Journal of Population Economics 6: 353-362.

Greenwood, J., Seshadri, A. and Vandenbroucke, G. (2005). The baby boom and baby bust. American Economic Review 95(1): 183-207.

Michel, P. and Wigniolle, B. (2007). On efficient child making, Economic Theory 31: 307-326.

Razin, A., and Ben-Zion, U. (1975). An intergenerational model of population growth. American Economic Review 65(5): 923-33.

Willis, R. (1973). A new approach to the economic theory of fertility behavior. Journal of Political Economy 81(2): S14-S64. 